\title{
Short stature-deafness-neutrophil dysfunction-dysmorphism syndrome
}

INSERM

\section{Source}

INSERM. (1999). Orphanet: an online rare disease and orphan drug data base. Short stature-deafness-neutrophil dysfunction-dysmorphism syndrome. ORPHA:2866

Short stature-deafness-neutrophil dysfunction-dysmorphism syndrome is characterised by short stature, sensorineural deafness, mutism, facial dysmorphism and abnormal neutrophil chemotaxis (leading to recurrent infections). 